\title{
Statistical Evaluation of Complex Sound Environment with Background Noise
}

\author{
Akira Ikuta and Hisako Orimoto \\ Department of Management Information Systems, Prefectural University of Hiroshima, 1-1-71 Ujina-Higashi, \\ Minamiku, Hiroshima 734-8558, Japan
}

\author{
Nazmul H. Siddique and Liam Philip Maguire \\ School of Computing and Intelligent Systems, University of Ulster, Northland Road, Londonderry BT48 $7 J L$, \\ United Kingdom
}

\begin{abstract}
(Received 21 January 2013; revised 6 December 2013; accepted 7 May 2014)
In order to evaluate noise in a sound environment, it is necessary to estimate the sound levels at evaluation points based on the observations at a reference point. In this study, a method is derived based on the observations contaminated by a background noise to estimate system parameters reflecting several orders of correlation information between the evaluation and reference points in a complex sound environment. Furthermore, a statistical evaluation method for traffic noise under the existence of background noise is proposed. The effectiveness of the proposed method is experimentally confirmed by applying it to the traffic noise data measured in a complex sound environment.
\end{abstract}

\section{INTRODUCTION}

In the evaluation of a sound environment around a main line, it is necessary to estimate the sound levels at evaluation points based on the observations at a reference point from the viewpoint of establishing a monitor system for the actual complex sound environment. Furthermore, the internal physical mechanism of an actual sound environment is often difficult to recognize analytically, and it contains unknown structural characteristics. In a previous study, ${ }^{1}$ it was found that complex sound environment systems are difficult to analyse by using usual structural methods based on the physical mechanism. Therefore, a nonlinear system model was derived in the expansion series form reflecting various types of correlation information from the lower order to the higher order between input and output variables. ${ }^{1}$ The conditional probability density function contains the linear and nonlinear correlations in the expansion coefficients, and these correlations play an important role as the statistical information for the input and output relationships of sound environment systems.

On the other hand, the random noise in an actual sound environment usually exhibits multifarious and complex characteristics such as non-Gaussian distribution and non-linear and non-stationary properties relating to natural, social, and/or human factors. Furthermore, the observation data are usually contaminated by background noise with complex statistical properties. In this situation, in order to evaluate the sound environment, precise estimation of the system characteristics of the sound environment is required, considering the contaminated observed data.

In this study, a general type of complex sound environment is considered. An estimation method for the sound levels at evaluation points for the complex sound environment around a main line such as a highway and a railroad is proposed on the basis of the observations at a reference point under the existence of background noise. By adopting an expansion expression of the conditional probability distribution as the system characteristics, a method to estimate the system parameters reflecting several orders of correlation information between the evaluation and reference points is first derived. Furthermore, a prediction method for the probability distribution of traffic noise at the evaluation points is also considered.

The effectiveness of the proposed theory is experimentally confirmed by applying it to actual data of road-traffic noise measured around a national road in the city of Hiroshima and low-frequency noise observed in a complicated sound environment near the exit of a tunnel, which is generated by Shinkansen trains running through the tunnel.

\section{EVALUATION OF TRAFFIC NOISE UNDER THE EXISTENCE OF BACKGROUND NOISE}

\subsection{Statistical Model for Sound Environment around a Main Line}

In the evaluation of traffic noise in a sound environment around a main line such as a highway and a railroad, it is necessary to estimate the sound levels at multiple evaluation points based on the observation at a reference point because of the difficulties of monitoring the sound levels at all evaluation points and at every instantaneous time. Furthermore, in the measurement of the sound environment, the observation data are generally contaminated by background noise.

The intensity variables satisfying the additive property of the specific noise and the background noise are considered in this section. Let $x$ and $y$ be the sound intensities of a specific noise at an evaluation point and a reference point, respectively. The probability distribution of $x$ has to be predicted on the basis of the observed data of $y$. Though the single evaluation point is considered, theoretically, for the simplification of the mathematical expression, the extension of the theory to the case of the multi-evaluation points is easy by considering multi-dimensional variable $\mathrm{x}$ instead of the single variable $x$. 